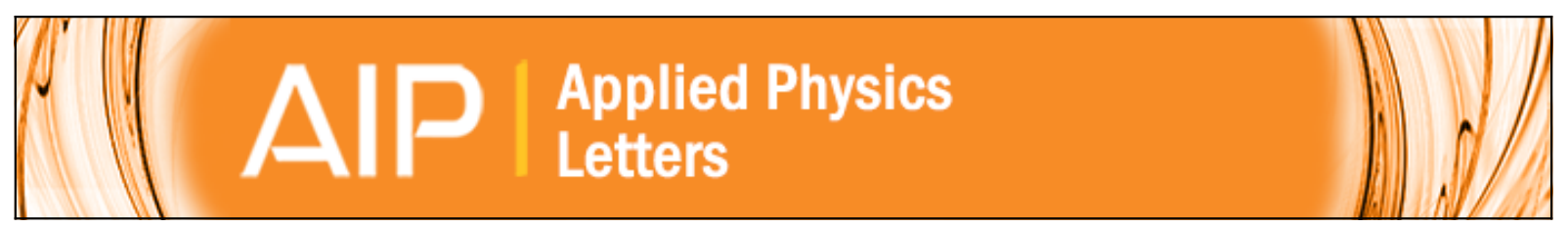

\title{
Rapid characterization of microscopic two-level systems using Landau-Zener transitions in a superconducting qubit
}

Xinsheng Tan, Haifeng Yu, Yang Yu, and Siyuan Han

Citation: Applied Physics Letters 107, 102601 (2015); doi: 10.1063/1.4930201

View online: http://dx.doi.org/10.1063/1.4930201

View Table of Contents: http://scitation.aip.org/content/aip/journal/apl/107/10?ver=pdfcov

Published by the AIP Publishing

\section{Articles you may be interested in}

Cavity quantum electrodynamics using a near-resonance two-level system: Emergence of the Glauber state Appl. Phys. Lett. 106, 172601 (2015); 10.1063/1.4918775

Probing the density of states of two-level tunneling systems in silicon oxide films using superconducting lumped element resonators

Appl. Phys. Lett. 106, 022603 (2015); 10.1063/1.4905149

Low-frequency Landau-Zener-Stückelberg interference in dissipative superconducting qubits

Low Temp. Phys. 39, 665 (2013); 10.1063/1.4818631

Dynamic behavior of Josephson-junction qubits: crossover between Rabi oscillations and Landau-Zener transitions

Low Temp. Phys. 31, 569 (2005); 10.1063/1.2001634

On the Landau-Zener formula for two-level systems

J. Math. Phys. 34, 2807 (1993); 10.1063/1.530097

\section{AIP $\left.\right|_{\text {APL Photonics }}$}

APL Photonics is pleased to announce Benjamin Eggleton as its Editor-in-Chief

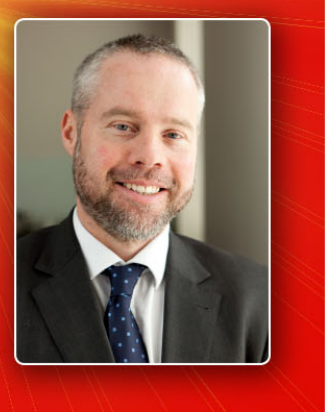




\title{
Rapid characterization of microscopic two-level systems using Landau-Zener transitions in a superconducting qubit
}

\author{
Xinsheng Tan, ${ }^{1,2}$ Haifeng $\mathrm{Yu},{ }^{1,3, a)}$ Yang $\mathrm{Yu},{ }^{1,3, b)}$ and Siyuan $\mathrm{Han}^{2}$ \\ ${ }^{1}$ National Laboratory of Solid State Microstructures, School of Physics, Nanjing University, Nanjing 210093, \\ China \\ ${ }^{2}$ Department of Physics and Astronomy, University of Kansas, Lawrence, Kansas 66045, USA \\ ${ }^{3}$ Synergetic Innovation Center of Quantum Information and Quantum Physics, University of Science and \\ Technology of China, Hefei, Anhui 230026, China
}

(Received 31 May 2015; accepted 25 August 2015; published online 8 September 2015)

\begin{abstract}
We demonstrate a fast method to detect microscopic two-level systems in a superconducting phase qubit. By monitoring the population leak after sweeping the qubit bias flux, we are able to measure the two-level systems that are coupled with the qubit. Compared with the traditional method that detects two-level systems by energy spectroscopy, our method is faster and more sensitive. This method supplies a useful tool to investigate two-level systems in solid-state qubits. (C) 2015 AIP Publishing LLC. [http://dx.doi.org/10.1063/1.4930201]
\end{abstract}

Superconducting qubits are promising candidates to realize quantum computation due to their scalability and design flexibility. ${ }^{1-4}$ However, in order to realize practical quantum computation, two crucial qubit parameters should be improved. One is the decoherence time and the other is gate fidelity. Recent investigations found that the microscopic two-level system (TLS) is one of the sources of energy relaxation, which sets a constraint on qubit decoherence time. TLSs are also harmful to Rabi oscillations, leading to a sharp decrease in gate fidelity. ${ }^{5-12}$ Since TLSs are ubiquitous in solid-state devices, ${ }^{13-15}$ it is important to detect and investigate them in order to achieve better qubit performance. ${ }^{6,16,17}$ In recent experiments on the mechanism and interaction of TLSs in superconducting qubits, TLSs are detected through the standard energy spectroscopy measurement. ${ }^{20,21}$ With this traditional method, the coupling between a TLS and a qubit results in an anti-crossing (or energy level splitting) on spectroscopy. ${ }^{5,6,18,19,22,23}$ However, the visibility of the anti-crossing depends on the width of the resonant peak as well as the measurement resolution. ${ }^{24}$ Timeconsuming high resolution spectroscopy measurement hinders the investigation of the dynamic evolution of TLSs in solid-state devices. Therefore, it is useful to develop a rapid measurement method to detect TLSs quickly and accurately.

Here, we propose and then experimentally demonstrate a rapid method to detect TLSs. In a flux bias superconducting phase qubit, we first excite the qubit to the excite state. We then sweep the flux bias in a range of $\delta \Phi$ with different speeds. If there is a splitting resulting from the coupling of the TLS and qubit in the region, Landau-Zener transition will occur. The population transferred to the other state depends on the coupling strength and sweeping speed. By measuring the population on the excited state after the sweeping as a function of the sweeping time, we can obtain accurate information on the TLS. Compared with the traditional method of spectroscopy, our method possesses higher

\footnotetext{
${ }^{a)}$ Electronic mail: hfyu@nju.edu.cn

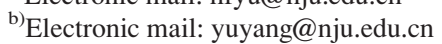

sensitivity and speed, supplying an alternative technique to investigate two-level systems in solid-state qubits.

A flux bias superconducting phase qubit can be considered as an artificial atom. ${ }^{23,25,26}$ When initialized in the ground state, it will be excited to the excited state by absorbing resonant microwave photons. A transition peak with width dependent on the decoherence time can be observed. By gradually changing the flux bias and microwave frequency gradually, one can map out the energy spectroscopy of the qubit, as shown in Fig. 1. This is the traditional method to detect a TLS. Ideally, the resonant frequency should decrease smoothly with increasing flux bias due to the anharmonicity of the phase qubit. When the spectroscopy is interrupted by a splitting, it is an indication that there is a coupling between a TLS and a qubit. The center of the splitting reveals the intrinsic frequency of the TLS, while the amplitude of the splitting $\Delta$ is 2 times of the coupling strength. If the coupling between TLS and qubit is weak which leads to a tiny splitting, one has to increase the sweep resolution of flux bias and microwave frequency. With this traditional method, significant amount of time will be needed to scan a 2-dimentional spectroscopy with frequency resolution much smaller than the rate of decoherence.

Noticing that the presence of the TLS is always associated with the anti-crossing, we propose to detect TLSs using the principle of Landau-Zener transition. The schematic time profile of the measurement is shown in the upper right part of Fig. 1. If we want to detect TLSs between $\Phi_{i}(\sim 0.9708$ $\left.\Phi_{0}\right)$ and $\Phi_{f}\left(\sim 0.9786 \Phi_{0}\right)$, we biased the qubit at $\Phi_{i}$ and apply a $\pi$ pulse to excite the system to state $|1\rangle$. Then, in a time duration $t_{s}$, we sweep the flux bias to $\Phi_{f}$ with a constant energy varying speed $v_{b} \equiv d E / d t$. At the end of sweeping, the population in $|1\rangle P_{1}$ is measured. We then change $t_{s}$, repeat the procedure, and obtain the corresponding $P_{1}$. If there is no TLS between $\Phi_{i}$ and $\Phi_{f}$, the population leaks from $|1\rangle$ only by the energy relaxation. From $\Phi_{i}$ to $\Phi_{f}$, the energy level spacing of the qubit changes about $0.3 \mathrm{GHz}$. In a welldesigned measurement system, the energy relaxation rate will be kept constant during the whole procedure. This can 


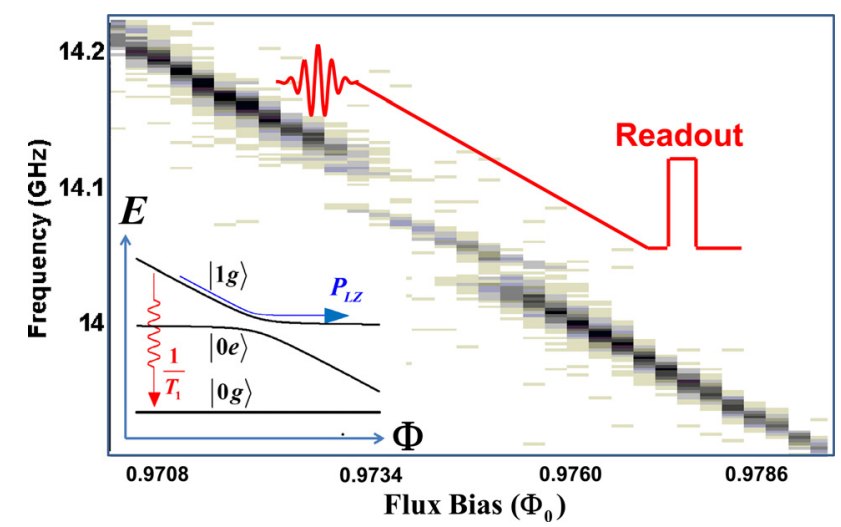

FIG. 1. Energy spectroscopy of a superconducting phase qubit. A large splitting resulting from the coupling between a TLS and a qubit can be clearly observed at flux bias about $0.9738 \Phi_{0}$. Upper right is the schematic time profile of the experimental procedure. Qubit is initially biased at $\Phi_{i}$ near 0.9708 $\Phi_{0}$. A $\pi$ pulse is applied to excite the qubit to $|1 g\rangle$. Then, the flux bias is swept to $\Phi_{f}$ with a constant speed. Finally, a readout pulse is sent to the qubit and the qubit state is measured. Left corner shows the schematic energy diagram of the TLS-qubit coupled system. The arrows indicate two channels of the population leaking from $|1 g\rangle$. One is the energy relaxation to $|0 g\rangle$ with a rate $1 / T_{1}$. The other is the Landau-Zener transition to $|0 e\rangle$ with a rate $P_{L Z \text {. }}$

be confirmed by directly measuring the energy relaxation time $T_{1}$ at various fixed flux biases. Therefore, if we plot $P_{1}$ as a function of $t_{s}$, we obtain an exponential decay with relaxation time $T_{1}$, which is the energy relaxation time of the qubit. However, in the presence of the TLS, there is another leak channel. The energy level diagram is schematically shown in the left corner of Fig. 1. The energy levels in the TLS-qubit coupled basis can be written as $|0 g\rangle,|1 g\rangle$, and $|0 e\rangle$, respectively, where $|0\rangle(|1\rangle)$ is the state of the qubit and $|g\rangle(|e\rangle)$ is the state of the TLS. The resonant $\pi$ pulse excites the system from $|0 g\rangle$ to state $|1 g\rangle$. When we sweep the flux bias across the anti-crossing, the qubit has finite probability to be transferred to state $|0 e\rangle$ through Landau-Zener transition. The transition probability is approximately given by Landau-Zener formula $P_{L Z}=\exp \left(-2 \pi \Delta^{2} / 4 v_{b} \hbar\right)$, which depends on the amplitude of splitting $\Delta$ and the sweeping speed $v_{b}{ }^{27}$ Now the population after sweeping can be written as

$$
P_{1}=e^{-t_{s} / T_{1}} e^{-\frac{\pi \Delta^{2}}{2 b_{b} h}}=e^{-t_{s} / T_{1 e f f}},
$$

where $T_{\text {leff }}=T_{1} /\left(1+\frac{\pi T_{1} \Delta^{2}}{2 \delta E \hbar}\right)$ and $\delta E$ is the energy difference of state $|1 g\rangle$ at $\Phi_{i}$ and $\Phi_{f}$. Therefore, having measured $T_{1}$ and $T_{1 \text { eff, }}$, we can detect the TLS and derive the amplitude of anticrossing. In our system, $T_{1}$ is fairly constant over the sweep range, which is proved by the previous measurement results.

Shown in Figs. 2(a) and 2(b) are some examples of $P_{1}$ vs. $t_{s}$ for various $\Phi_{f}$. In our experiments, we set $\Phi_{i}=0.9708 \Phi_{0}$. $\Phi_{f}$ ranges from $0.9708 \Phi_{0}$ to $0.9786 \Phi_{0}$. The energy difference $\delta E$ for $0.9708 \Phi_{0}$ and $0.9786 \Phi_{0}$ is $\delta E / 2 \pi=300 \mathrm{MHz}$, which is much larger than $\Delta / 2 \pi \sim 50 \mathrm{MHz}$. In this range, the frequency of qubit is linear in flux, which indicates that the constant varying speed of $v_{b} \equiv d E / d t$ is convenient to achieve. Moreover, for a large $\delta E$, we can always design the waveform of flux sweeping to keep a constant $v_{b}$ if we have known the qubit energy spacing as a function of the flux bias. Therefore,
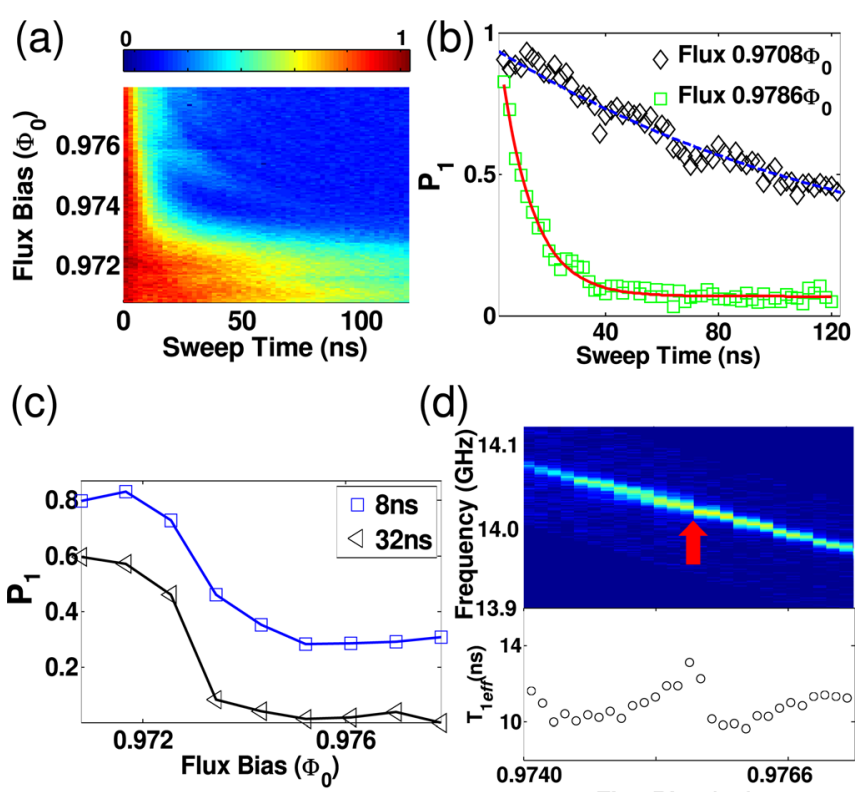

(d)

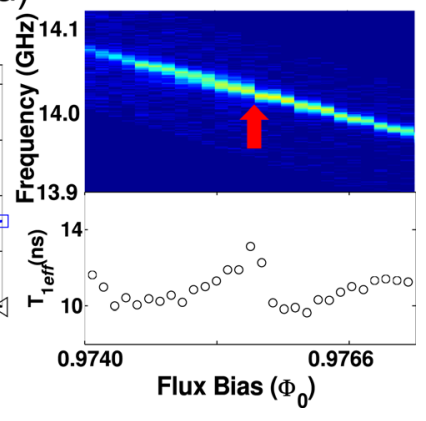

FIG. 2. (a) Population of $|1 g\rangle$ as a function of sweeping time $t_{s}$ for various final flux biases $\Phi_{f}$. (b) Effective energy relaxation time determined from the population in $|1 g\rangle$ vs. sweeping time $t_{s}$ for two final flux biases $\Phi_{f}=0.9708$ $\Phi_{0}$ (square) and $0.9786 \Phi_{0}$ (diamond). By fitting the curve to an exponential decay, we can obtain $T_{1 \text { eff. }}$ Since $\Phi_{f}=0.9708 \Phi_{0}, T_{1 \text { eff }}$ obtained from the upper curve is identical to $T_{1}$. (c) $P_{1}$ vs. final flux bias for two sweeping time. The line is a guide to the eye. A quick decrease of $P_{1}$ near $0.9734 \Phi_{0}$ indicates the presence of a TLS. (d) Upper panel: Spectroscopy of the qubit for flux bias from $0.9740 \Phi_{0}$ to $0.9773 \Phi_{0}$. No obvious splitting can be observed. Lower panel: $T_{1 \text { eff }}$ vs. final flux bias in the same range. A discontinuity at $0.9757 \Phi_{0}$ can be clearly seen. The red arrow marks the corresponding position of a small TLS on spectroscopy.

Eq. (1) is, in general, a good approximation. We can see that with the increase in $\Phi_{f}$, the sweeping range hits the TLS and a decrease in $T_{1 \text { eff }}$ could be observed. We have measured the stationary $T_{1}$ at various flux biases without sweeping the flux bias. $T_{1}=140 \pm 4 \mathrm{~ns}$ and does not change with flux bias. By fitting $P_{1}$ vs. $t_{s}$ in Fig. $2(\mathrm{~b})$, we obtain $T_{1 \text { eff }}=11.00 \pm 0.01 \mathrm{~ns}$ for $\Phi_{f}=0.9786 \Phi_{0}$. Using the definition of $T_{1 \text { eff, }}$, we obtain $\Delta / 2 \pi=48.3 \pm 2.5 \mathrm{MHz}$, which agrees with the observation in the spectroscopy.

A comparison between our method with the traditional method demonstrates the advantage of the former in terms of resolution and speed. For the spectroscopy method, the resolution is determined by the width of resonant peak, which is the inverse of $T_{1}$ without considering the dephasing. Using measured $T_{1}$, the smallest TLS we can detect with spectroscopy is about $7 \mathrm{MHz}$ for our sample. In order to measure a spectroscopy of a $50 \mathrm{MHz}$ TLS, we have to scan a window of $100 \mathrm{MHz}$ frequency (with $1 \mathrm{MHz}$ step) and $8 \mathrm{~m} \Phi_{0}$ flux bias (with $0.2 \mathrm{~m} \Phi_{0}$ step). The total points we need to measure is about 4000 in the window. On the other hand, the resolution of our method is determined by the uncertainty of $T_{1 \text { eff. }}$ The largest uncertainty is achieved when $T_{1 \text { eff }}$ approaches $T_{1}$ because the smaller the TLS, the larger the

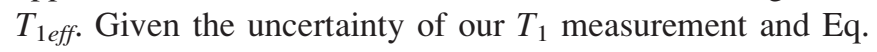
(1), the smallest $\Delta$ we can detect is about $6 \mathrm{MHz}$, which is slightly smaller than that of the spectroscopy method. However, we only need to measure about 50 points to obtain 
$T_{1 \text { eff, }}$ which is about 2 order of magnitude faster than the spectroscopy measurement.

Therefore, an obvious advantage of our measurement technique is the quick detection of the presence of the TLS. There is a room to further shorten the measurement time. We can just compare the $P_{1}$ instead of $T_{1 \text { eff }}$ so we do not need to change the sweeping time and repeat the measurement procedure. Shown in Fig. 2(c) are examples of $P_{1}$ vs. sweeping distance for two different sweeping speeds. We decrease the measurement points to about 10. A fast decrease of $P_{1}$ near $0.9727 \Phi_{0}$ reflects a large TLS presence on spectroscopy. Therefore, if we have already known $T_{1}$, we can monitor a TLS in real time by detecting $P_{1}$ while sweeping the flux bias. Although we could not extract the exact value of $\Delta$ by this means, it will tell us the relative amplitude of the coupling. This is very powerful for the investigation of the dynamics of TLS.

The high sensitivity of our method can be demonstrated by measuring small TLSs. For instance, in Fig. 2(d) upper panel, there is a small TLS which can hardly be observed from spectroscopy. However, if we measure $T_{1 e f f}$ for different flux biases, a discontinuity at $0.9757 \Phi_{0}$ can be found, indicating that there is a small TLS at that flux bias.

We noticed that Eq. (1) is an asymptotic formula for Landau-Zener transition. ${ }^{27}$ It is an exact solution only under the condition of sweeping flux bias from $-\infty$ to $+\infty$. In order to precisely determine $\Delta$, we have to carefully check the validity of the approximation. For the finite sweeping range, we can use the master equation to describe the dynamics of a qubit. Considering the effect of decoherence, we can write the master equation as

$$
\dot{\rho}=-i[H, \rho]-\Gamma[\rho],
$$

where

$$
H=\left[\begin{array}{cc}
\omega_{01}(t) & \frac{\Delta}{2} \\
\frac{\Delta}{2} & \omega_{T L S}
\end{array}\right] .
$$

Here, $\omega_{01}(t)=\omega_{01}(0)-v_{b} t$ is the energy level spacing of the qubit and $\omega_{T L S}$ is the intrinsic frequency of the TLS. The second term $\Gamma[\rho]$ describes the relaxation and dephasing processes phenomenologically. In a concrete expression, Eq. (2) can be rewritten as

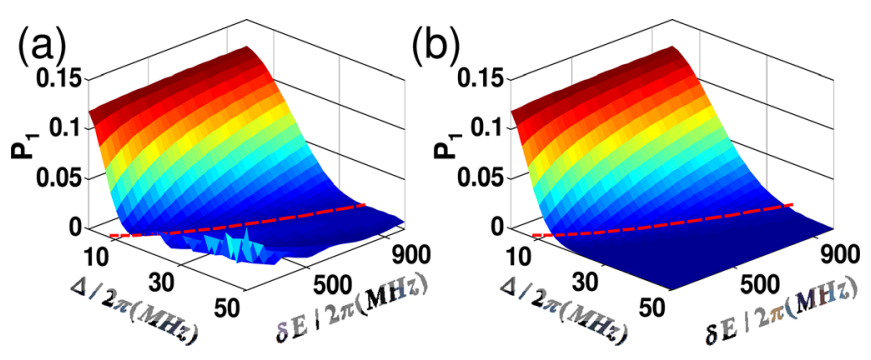

FIG. 3. Simulation results of $P_{1}$ as a function of different sweeping energy $\delta E$ and splitting $\Delta$ by using master equations (a) and analytic Landau-Zener transition rate (b). The dashed lines in both figures represent $\Delta^{2} T / \delta E=0.2$, in which $T$ is sweep time. When $\Delta^{2} T / \delta E<0.2$, the Landau-Zener asymptotic formula is a good approximation. Therefore, in this region, our method can accurately obtain the gap value.

$$
\begin{aligned}
& \dot{\rho}_{a a}=-i\left[\frac{\Delta}{2}\left(\rho_{b a}-\rho_{a b}\right)\right]-\Gamma_{a} \rho_{a a} \\
& \dot{\rho}_{b b}=i\left[\frac{\Delta}{2}\left(\rho_{b a}-\rho_{a b}\right)\right]-\Gamma_{b} \rho_{b b} \\
& \dot{\rho}_{a b}=-i\left[\left(\omega_{01}(t)-\omega_{T L S}\right) \rho_{a b}+\frac{\Delta}{2}\left(\rho_{b b}-\rho_{a a}\right)\right]-\gamma \rho_{a b},
\end{aligned}
$$

with $\rho_{b a}=\rho_{a b}^{\star}$. Here, $|1 g\rangle$ and $|0 e\rangle$ are labeled as $|a\rangle$ and $|b\rangle . \Gamma_{a}$ and $\Gamma_{b}$ are the energy relaxation rate to $|0 g\rangle$ from $|1 g\rangle$ and $|0 e\rangle$, respectively. $\gamma=\left(\Gamma_{a}+\Gamma_{b}\right) / 2+1 / T_{2}$ is the decoherence rate including both energy relaxation and phase dephasing. In Fig. 3, we compare the results obtained from numerical solution of the master equation and those obtained from Eq. (1). The two results generally agree well, except for when $\Delta^{2} T>0.2 \delta E$, in which $T$ is sweep time. Therefore, in order to obtain accurate $\Delta$ value, one should maximize $\delta E / T$, which is the sweeping speed.

There are two more issues we should mention for our method. One is that our method assumes that $T_{1}$ does not change during the sweeping. This may not be valid in some systems, which will make it difficult to obtain an accurate value of $\Delta$. Nevertheless, if one knows the background of $T_{1}$ as a function of the energy detuning, one can use our method to detect the presence and relative magnitude of a TLS. The other issue is the presence of multiple TLSs with the increase of $\delta E$. When the distance between TLSs $\delta E$ is larger than $\Delta$, one can use our method to characterize the TLSs one by one. If the distance between TLSs is small, one can replace $\Delta$ in Eq. (1) with $\sum \Delta_{i}$, which is an effective energy gap of the whole system. In this case, our measurement will obtain the property of the whole TLSs instead of individual TLSs.

In summary, we have demonstrated a rapid method to detect TLSs in superconducting phase qubits. By sweeping the qubit bias flux, we measure the effective relaxation time, from which we can measure the TLS that coupled with the qubit. Compared with the traditional method of spectroscopy, the method can detect TLSs with higher resolution and speed, thus supplying a useful tool to characterize two-level systems in solid-state qubits. ${ }^{28}$

This work was partially supported by the State Key Program for Basic Research of China SKPBR of China (2011CB922104 and 2011CBA00205) and NSFC (91321310, 11274156,11504165 , and 11474152).

${ }^{1}$ Y. Makhlin, G. Schön, and A. Shnirman, Rev. Mod. Phys. 73, 357 (2001).

${ }^{2}$ J. You and F. Nori, Phys. Today 58(11), 42 (2005).

${ }^{3}$ J. Clarke and F. K. Wilhelm, Nature 453, 1031 (2008).

${ }^{4}$ M. H. Devoret and R. J. Schoelkopf, Science 339, 1169 (2013).

${ }^{5}$ R. W. Simmonds, K. M. Lang, D. A. Hite, S. Nam, D. P. Pappas, and J. M. Martinis, Phys. Rev. Lett. 93, 077003 (2004).

${ }^{6}$ J. M. Martinis, K. B. Cooper, R. McDermott, M. Steffen, M. Ansmann, K. D. Osborn, K. Cicak, S. Oh, D. P. Pappas, R. W. Simmonds, and C. C. Yu, Phys. Rev. Lett. 95, 210503 (2005).

${ }^{7}$ J. Gao, M. Daal, J. M. Martinis, B. A. Mazin, P. K. Day, H. G. Leduc, A. Vayonakis, B. Sadoulet, and J. Zmuidzinas, Appl. Phys. Lett. 92, 212504 (2008).

${ }^{8}$ S. Ashhab, J. R. Johansson, and F. Nori, New J. Phys. 8, 103 (2006).

${ }^{9}$ A. Shnirman, G. Schön, I. Martin, and Y. Makhlin, Phys. Rev. Lett. 94, 127002 (2005).

${ }^{10}$ F. Meier and D. Loss, Phys. Rev. B 71, 094519 (2005).

${ }^{11}$ M. Bhattacharya, K. D. Osborn, and A. Mizel, Phys. Rev. B 84, 104517 (2011). 
${ }^{12}$ L. C. Ku and C. C. Yu, Phys. Rev. B 72, 024526 (2005).

${ }^{13}$ G. Zolfagharkhani, A. Gaidarzhy, S. Shim, R. L. Badzey, and P. Mohanty, Phys. Rev. B 72, 224101 (2005).

${ }^{14}$ F. Hoehne, Yu. A. Pashkin, O. Astafiev, L. Faoro, L. B. Ioffe, Y. Nakamura, and J. S. Tsai, Phys. Rev. B 81, 184112 (2010).

${ }^{15}$ O. Arcizet, R. Riviere, A. Schliesser, G. Anetsberger, and T. J. Kippenberg, Phys. Rev. A 80, 021803 (2009).

${ }^{16}$ I. Martin, L. Bulaevskii, and A. Shnirman, Phys. Rev. Lett. 95, 127002 (2005).

${ }^{17}$ Y. Shalibo, Y. Rofe, D. Shwa, F. Zeides, M. Neeley, J. M. Martinis, and N. Katz, Phys. Rev. Lett. 105, 177001 (2010).

${ }^{18}$ A. Lupaşcu, P. Bertet, E. F. C. Driessen, C. J. P. M. Harmans, and J. E. Mooij, Phys. Rev. B 80, 172506 (2009).

${ }^{19}$ J. Lisenfeld, C. Müller, J. H. Cole, P. Bushev, A. Lukashenko, A. Shnirman, and A. V. Ustinov, Phys. Rev. B 81, 100511(R) (2010).

${ }^{20}$ G. J. Grabovskij, T. Peichl, J. Lisenfeld, G. Weiss, and A. V. Ustinov, Science 338, 232 (2012).
${ }^{21}$ J. Lisenfeld, G. J. Grabovskij, C. Müller, J. H. Cole, G. Weiss, and A. V. Ustinov, Nat. Commun. 6, 6182 (2015).

${ }^{22}$ T. A. Palomaki, S. K. Dutta, R. M. Lewis, A. J. Przybysz, H. Paik, B. K. Cooper, H. Kwon, J. R. Anderson, C. J. Lobb, F. C. Wellstood, and E. Tiesinga, Phys. Rev. B 81, 144503 (2010).

${ }^{23}$ G. Sun, X. Wen, B. Mao, J. Chen, Y. Yu, P. Wu, and S. Han, Nat. Commun. 1, 51 (2010).

${ }^{24}$ M. J. A. Stoutimore, M. S. Khalil, C. J. Lobb, and K. D. Osborn, Appl. Phys. Lett. 101, 062602 (2012).

${ }^{25}$ R. McDermott, R. W. Simmonds, M. Steffen, K. B. Cooper, K. Cicak, K. D. Osborn, S. Oh, D. P. Pappas, and J. M. Martinis, Science 307, 1299 (2005).

${ }^{26}$ X. Tan, D. Zhang, Z. Zhang, Y. Yu, S. Han, and S. Zhu, Phys. Rev. Lett. 112, 027001 (2014).

${ }^{27}$ S. Shevchenko, S. Ashhab, and F. Nori, Phys. Rep. 492, 1 (2010).

${ }^{28}$ G. Ithier, E. Collin, P. Joyez, P. J. Meeson, D. Vion, D. Esteve, F. Chiarello, A. Shnirman, Y. Makhlin, J. Schriefl, and G. Schön, Phys. Rev. B 72, 134519 (2005). 